\title{
Estimation of Fatigue Life for U-Shaped Metal Expansion Bellows
}

\author{
S. K. Makke, A. A. Keste, S. H. Gawande \\ (Department of Mechanical Engineering, M. E. S. College of Engineering, Pune, S.P.Pune University, India)
}

\begin{abstract}
Bellows is a standout amongst the most vital segment in the expansion joint and has the capacity to absorb regular and irregular expansion and contraction of the system. Bellows requires high strength and great flexibility, which can be accomplished by great design and appropriate manufacturing strategy. The design is referred from EJMA (Expansion Joint Manufacturing Association) yet at the same time proper arrangement choice makes it troublesome The metal bellows are fabricated with various techniques like shaping, hydro forming, bulging, drawing, deep drawing, which relies up on application. Expansion joints are intended to operate with a value for deflection stress that exceeds the yield strength of the bellows material. This implies most development joints will take a permanent set at rated axial, angular or lateral movement. Expansion joint bellows are rarely designed to work in the elastic stress range. Therefore the bellows will eventually fatigue after a limited number of movement cycles. It is vital to indicate a practical cycle life as an design consideration when requesting an expansion joint. In this work we will perform study finite number of cycles taken by the bellow of the selected application using analytical and simulation approach. At the same time parametric study will be performed to optimize the bellow design. Actual testing will be performed in the end and results of the actual testing will be compared with the simulation and analytical solution results.
\end{abstract}

Keywords - Analytical approach; Bellows; Fatigue life analysis; Membrane stress; MATLAB Simulation.

\section{INTRODUCTION}

Metal expansion joints (additionally called compensators) are repaying components for thermal expansion and relative development in pipelines, compartments and machines. They comprise of at least one metal bellows, connectors at both finishes, and tie poles that depend on the application. They are separated by the three essential sorts of development: axial, angular and extension joints. Restricted measure of research work has been done by a few scientists working in the region of the expansion joint for shell and tube heat exchanger. Their work has been accounted for by performing mechanical overview (to be specific, Alfa Laval India Ltd., Pune) and thorough writing audit through prior distributed research work, journal papers, and specialized reports. Many plan formulae of bellows can be found in ASME code [1]. And the most comprehensive and broadly acknowledged content on bellows configuration is the Guidelines of Expansion Joint Manufactures Association, EJMA [2]. Number of pilot and test tests have been performed for investigation of AM350 steel bellows by Shaikh et al. [3]. As bellows are presented to marine air for over 13 years which prompts to setting impact, henceforth the assurance of element qualities of bar limited components by controlling certain parameters on business programming was finished by Broman et al. [4]. In correlation with semi investigative, techniques have capability of considering axial, twisting, and torsion degrees of freedom in the meantime, and the rest are demonstrated by limited components in which experimental outcomes are additionally checked. The impact of the elliptic level of $\Omega$-formed bellows toroid on its stresses is researched by Li [5]. Also, Becht IV [6] has explored the fatigue behavior of expansion joint bellows. The after effects of $\Omega$-formed bellows with elliptic toroid computed stresses relate to tests. The elliptic level of $\Omega$-molded toroid influences the magnitude of internal pressure-induced stress and axial deflection-induced stress. It particularly creates an extensive impact on the pressure-induced stress. To keep up the weariness life of toroid bellows, during assembling process toroid elliptic degree must be diminished. EJMA stresses for unreinforced bellows are assessed by Becht IV [6]. Utilizing direct axisymmetric shell components parametric examination is led. Finite element analysis is done using commercial code. Meridional stresses because of internal pressure and displacement are exact. bellows shaping procedure is done in the wake of assessing powerful parameters by Faraji et al. [7]. FEM results are compared with analytical analysis. Faraji et al. [8] utilized a commercial FEM code, ABAQUS Explicit, to reproduce fabricating procedure of metal bellows. Forming of various states of tubular bellows utilizing a hydroforming procedure is proposed by Kang et al. [9].

A reasonable blend of hydroforming and roll-forming forms which is analyzed as a feasible contrasting option to improve the fatigue life of austenitic steel bellows for low-pressure, low-temperature applications. This blend procedure has the additional merits that bellows with additional deep convolutions can be formed as substitutions to costlier diaphragm type bellows and for applications having space restrictions. Examination uncovers that a relationship exists between this unequal sharing of development and fatigue life. In view of 6th National Conference RDME 2017, 17th-18th March 2017. 
these perceptions another non-destructive extend test is proposed with which unique life evaluations can be refined proposed by Subramanian et al. [10]. Weariness is for the most part an essential part of design on metallic bellows expansion joints. These parts are liable to relocation stacking which oftentimes brings about cyclic strains. This review has been examined to investigate the impact of geometry on fatigue life for automotive bellows. 8 node shell component and non-direct technique is utilized for the investigation. The advanced states of the bellows are required to give great rules to the useful designs proposed by Jinbong et al.[11]. Experimentally the toroid-molded bellows conduct. The outcomes demonstrated that, contrasted with Ushaped bellows, toroidal bellows have longer exhaustion life, more grounded capacity to oppose internal pressure instability proposed by Tingxin et al. [12]. The impact of natural medium on erosion fatigue life for Ushaped bellows expansion joints. The outcomes demonstrated that the presence of destructive medium accelerates both split start and propagation rates and diminishes the failure life for the expansion joints proposed by Zhu et al. [13]. The failure of a metal bellows adaptable hose which subject to numerous pressure cycles. It was found that by cycling the pressure on the hose and applying a pressure significantly greater than the yield capacities of the hose, this blend of yielding and cycling, brought about a burst failure proposed by Penetrate and Evans et al. [14]. used acoustic outflow method to monitor crack progressions of a shaped $300 \mathrm{~mm}$ long stainless steel bellows subjected to cyclic axial stacking with vibration amplitude of $15 \mathrm{~mm}$. AEs from fatigue cracks in $0.12 \mathrm{~mm}$ thick bellow with 10 convolutions were monitored by three resonant type AE sensors mounted on the flange weld to the bellow ends. The bellows failed at 26770 cycles after producing three circumferential cracks near the upper jig and lower flange. First AE was detected at 21782 cycles. This timing was 1500 cycles earlier than the first circumferential crack near the upper jig observed by video camera. AE count increased after 24000 cycles at which large third circumferential cracks were produced near the lower flange proposed by Koji K. et al. [15]. Emil Witzenmann was viewed as the innovator of expansion joints. In 1920, he connected for a patent for the primary purported adaptable metal tube extension joint, German Reichspatent No. 367 185, from 29 July 1920. From a technical point of view, this antecedent of today's expansion joints is a substantial, pressure tight adaptable metal hose with a defined, limited opportunity of development. In the 1930s, the 'metal hose' or 'adaptable metal tube' principle was replaced by the metal bellows as the focal useful component. This design standard - metal bellows with association pieces - is still the basic of modern metal expansion joints today. In 1898 In 1898 A patent was connected for "flexible metal tubes with beading folds" (convolutions), Creation of bellows and metal hoses of consistent layered tubes for mechanical applications, was therefore first ever thusly maker by, the organization now known as, The BOA Group.

\section{PROBLEM FORMULATION AND OBJECTIVE}

According to the literature and modern Industrial survey, it is seen that Bellow is a standout amongst the most vital component in the expansion joint and has the capacity to absorb regular and irregular expansion and contraction of the system. expansion joints are intended to work with a value for deflection stress that far surpasses the yield strength of the bellows material. This implies most expansion joints will take a permanent set at the rated axial, angular or lateral movement. Expansion joint bellows are designed to operate in the elastic stress range. In this way the bellows will eventually fatigue after a limited number of movement cycles. It is essential to indicate a practical cycle life as a design consideration when requesting an expansion joint. An excessively conservative cycle life necessity can result in a bellows design that is so long and delicate that it is liable to squirm failure.

\section{ANALYTICAL ANALYSIS}

Metal expansion bellows are an exceptionally particular segment of a funneling framework. They should be designed sufficiently strong enough to accommodate the framework design pressure and also sufficiently adaptable to accept the design deflections for a figured number of events, with a minimum resistive force. So as to understand the static and dynamic behavior of metal expansion bellows. it is important to concentrate the choice of materials of bellows for the given application, essential thing, their appropriate design, and working. The different mechanical properties and design parameters for bellows under consideration are shown in Table1.

\subsection{Determination of fatigue life cycle:-}

1. Mean diameter of bellows convolutions $\left(\mathrm{D}_{\mathrm{m}}\right)$

$$
=D_{b}+w+n \times t
$$

2. Thickness of one ply, corrected for Thinning during forming $\left(t_{p}\right)$

$$
=t \times \sqrt{\frac{D_{b}}{D_{m}}}
$$

6th National Conference RDME 2017, 17th- 18th March 2017. 
3. Bellows cross sectional area of one convolution (A)

$$
=\left[\frac{(\pi-2) \times q}{2}+2 \times w\right] \times n \times t_{p}
$$

Table 1: Different design parameters

\begin{tabular}{|c|c|c|}
\hline Parameters & Symbol & Specifications/Values \\
\hline Expansion Joint Material & & SA-240 321 \\
\hline Material UNS Number & & S32100 \\
\hline Bellows Design Allowable Stress & $S$ & $129.65 \mathrm{~N} / \mathrm{mm}^{2}$ \\
\hline Bellows Ambient Allowable Stress & $S_{a}$ & $137.89 \mathrm{~N} / \mathrm{mm}^{2}$ \\
\hline Bellows Yield Stress & $S_{y}$ & $161.86 \mathrm{~N} / \mathrm{mm}^{2}$ \\
\hline Bellows Elastic Modulus at Design Temp. & $E b$ & $183090 \mathrm{~N} / \mathrm{mm}^{2}$ \\
\hline Bellows Elastic Modulus at Ambient Temp & $E_{O}$ & $195121 \mathrm{~N} / \mathrm{mm}^{2}$ \\
\hline Poisson's Ratio & $v_{b}$ & 0.300 \\
\hline Bellows Material Condition & & Formed \\
\hline Design Cycle Life, Required number of cycles & Nreq & 7000 \\
\hline Design Internal pressure & $P$ & $1.099 \mathrm{~N} / \mathrm{mm}^{2}$ \\
\hline Design temperature for Internal Pressure & & $190^{\circ} \mathrm{c}$ \\
\hline Bellow Type & & U- Shaped \\
\hline Bellows inside diameter & $D B$ & $131.000 \mathrm{~mm}$ \\
\hline Convolution Depth & $W$ & $8.000 \mathrm{~mm}$ \\
\hline Convolution Pitch & $Q$ & $8.000 \mathrm{~mm}$ \\
\hline Expansion joint opening per convolution & $\Delta Q$ & $0.2985 \mathrm{~mm}$ \\
\hline Total number of convolutions & $N$ & 10 \\
\hline Nominal thickness of one ply & $T$ & $0.300 \mathrm{~mm}$ \\
\hline Total number of plies & $N$ & 3 \\
\hline End tangent Length & $L T$ & $13.000 \mathrm{~mm}$ \\
\hline Fatigue Strength Reduction Factor & $K g$ & 1.500 \\
\hline
\end{tabular}

4. Stiffening effect factor $(\mathrm{k})$

5. Coefficient (C1)

$$
=\operatorname{MIN}\left[1.0, \frac{L_{1}}{\left[1.5 \times \sqrt{D_{b} \times t}\right]}\right]
$$

$$
=\frac{q}{2 \times w}
$$

6. Coefficient (C2)

$$
=\frac{q}{\left[2.2 \times \sqrt{D_{m} \times t_{p}}\right]}
$$

Table 2: Different design specification for 10 convolution bellows

\begin{tabular}{|l|l|l|}
\hline Specification & Symbol & Values \\
\hline Mean diameter of bellows convolutions & $\mathrm{D}_{\mathrm{m}}$ & $139.900 \mathrm{~mm}$ \\
\hline Thickness of one ply, corrected for Thinning during forming & $\mathrm{t}_{\mathrm{p}}$ & $0.290 \mathrm{~mm}$ \\
\hline Bellows cross sectional area of One convolution & $\mathrm{A}$ & $17.90 \mathrm{~mm}^{2}$ \\
\hline Stiffening effect factor & $\mathrm{K}$ & 1 \\
\hline Coefficient & $\mathrm{C} 1$ & 0.5 \\
\hline Coefficient & $\mathrm{C} 2$ & 0.571 \\
\hline
\end{tabular}

\subsection{Fatigue life calculation by analytical analysis:-}

The circumferential membrane stress $(S 1)$ in bellows tangent due to internal pressure is given as per $S 1=\frac{1}{2}\left\{\frac{L t \times E b \times K \times P \times(D b+n \times t)^{2}}{[n \times t \times(D b+n \times t) \times L t \times E b \times t c \times D c \times L c \times E c \times K]}\right\}$

The end convolution circumferential membrane stress (S2) due to internal pressure based on the equilibrium considerations is as shown in Figure 1. Equation (2) shows the end convolution circumferential membrane stress 
$S_{2, E}=\frac{1}{2}\left\{\frac{[q \times D m+L t \times(D b+n \times t)] \times P}{\left(A+n \times t_{p} \times L t+t_{c} \times L c\right)}\right\}$

Where, $D m$ is mean diameter of bellows convolution and it is given as,

$D m=D b+w+n \times t$

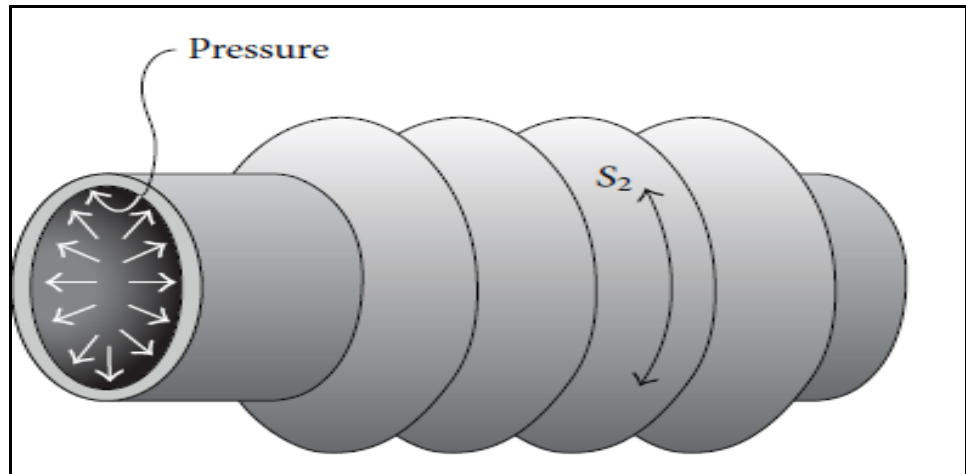

Fig1: Deflection stresses acting on bellows.

The intermediate convolution circumferential membrane stress $(S 2$,$) due to internal pressure is calculated by$ using the following equation (4).

$S_{2, I}=\frac{1}{2}\left\{\frac{P \times q \times D m}{A}\right\}$

The bellows meridional membrane stress $(S 3)$ due to internal pressure is calculated based on the component of pressure in axial direction acting on the convolution divided by the metal area of root and crown by using the following equation (5).

$S 3=\frac{1}{2}\left\{\frac{W \times P}{n \times t_{p}}\right\}$

The bellows meridional bending stress $(S 4)$ due to internal pressure as is given by equation (6).

$S 4=\left[\frac{1}{2 \times n}\right] \times\left[\frac{w}{t_{p}}\right]^{2} \times P \times C_{p}$

The bellows meridional membrane stress (S5) and meridional bending stress (S6) due to deflection are given by equation (7).

$S 5=E b \times\left(t_{p}\right)^{2} \times \frac{\Delta Q}{\left(2 \times w^{2} \times C f\right)}$

$S 6=5 \times E b \times t_{p} \times \frac{\Delta Q}{\left(3 \times w^{2} \times C d\right)}$

Where $C p, C f$, and $C d$ are the factors for calculating $S 4, S 5, S 6$ respectively.Eb is modulus of elasticity for bellows. Unreinforced total stress range due to cyclic displacement (St) are given by equation (8).

$$
\begin{aligned}
& \mathrm{St}=0.7 \times(S 3+S 4)+(S 5+S 6) \\
& \text { Kg Factor }=k g \times\left(E_{\mathrm{o}} / E_{b}\right) \times s t \\
& \text { For Kg Factor }<448 \mathrm{~N} / \mathrm{mm}^{2} \\
& \text { Ko }=46200.000 \\
& \text { So }=211.00 \mathrm{~N} / \mathrm{mm}^{2}
\end{aligned}
$$

Allowable number of Fatigue Cycles $\left(\mathrm{N}_{\text {allww }}\right)$ are given by equation (9).

$$
\text { Nallw }=\left[\frac{K_{o}}{K_{g}\left[\frac{E_{o}}{E_{b}}\right] S_{t}-S_{o}}\right]^{2}
$$


Table 3: Summary of analytical analysis for 10 convolution bellows

\begin{tabular}{|l|l|l|l|l|}
\hline Types of Stress & Analytical Stress & $\mathbf{0 . 2 M p a}$ \\
\hline & $\mathbf{0 . 0 5} \mathbf{M p a}$ & $\mathbf{0 . 1 M p a}$ & $\mathbf{0 . 1 5} \mathbf{M p a}$ & 0.919 \\
\hline S3 & 0.229 & 0.459 & 0.689 & 16.437 \\
\hline S4 & 4.109 & 8.218 & 12.328 & 2.739 \\
\hline S5 & 2.739 & 2.739 & 2.739 & 236.12 \\
\hline St & 236.12 & 236.12 & 236.12 & 251.008 \\
\hline Kg Factor & 241.895 & 244.932 & 247.97 & 401.252 \\
\hline$N_{\text {allw }}$ & 386.686 & 391.54 & 396.396 & 58969 \\
\hline & 69152 & 65484 & 62098 & \\
\hline
\end{tabular}

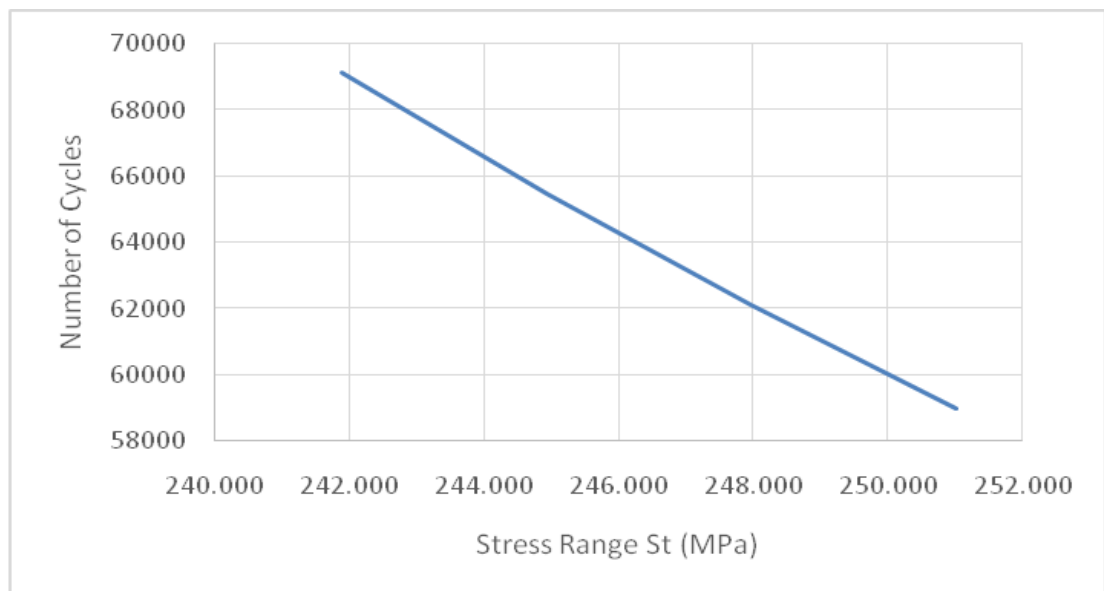

Fig 2:- S-N Curve using Analytical Solution from MS Excel

IV. SIMULATION IN MATLAB

Matlab Program Used for the Results Calculation of the Stresses and life cycles using conventional formulae and creating a program with for loops for pressures varying from $0.05 \mathrm{MPa}$ to $0.2 \mathrm{MPa}$. Program will give us values of all the Stresses and Kg Factor and Allowable cycles in single pass. Output of the MATLAB program is given in the table below.

Table 4:- MATLAB program Output

\begin{tabular}{|l|l|l|l|l|}
\hline Input & \multicolumn{4}{l|}{ Matlab Simulation Outputs } \\
\hline Pressure Applied & 0.05Mpa & 0.1Mpa & 0.15Mpa & 0.2Mpa \\
\hline$S 3$ & 0.230 & 0.459 & 0.689 & 0.919 \\
\hline$S 4$ & 4.114 & 8.227 & 12.341 & 16.454 \\
\hline$S 5$ & 2.776 & 2.776 & 2.776 & 2.776 \\
\hline$S 6$ & 236.096 & 236.096 & 236.096 & 236.096 \\
\hline St & 241.912 & 244.952 & 247.993 & 251.033 \\
\hline Kg Factor & 386.713 & 391.573 & 396.433 & 401.293 \\
\hline$N_{\text {allw }}$ & 69132 & 65461 & 62074 & 58944 \\
\hline
\end{tabular}

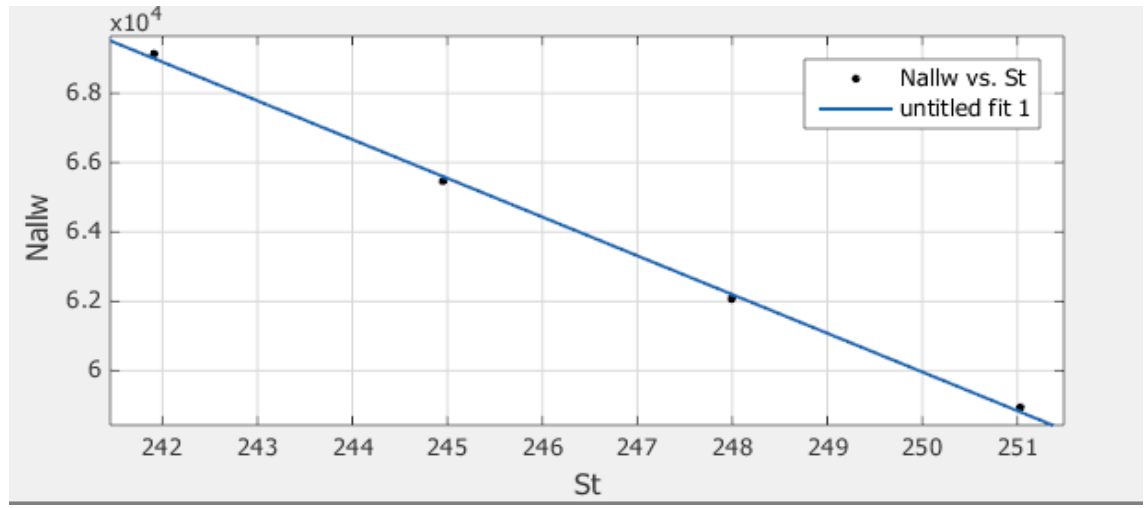

Fig 3:- S-N Curve by Matlab Simulation 


\section{RESULT S AND DISCUSSION}

Fig. 2 and Fig. 3 shows the S-N diagram and also Table 5 shows the comparison of the fatigue life obtained by analytical approach and MATLAB simulation approach. On comparison it is seen that fatigue life obtained by both approaches shows close match.

Table 5: Summary of fatigue life results

\begin{tabular}{|l|l|l|l|}
\hline $\begin{array}{l}\text { Sr. } \\
\text { No. }\end{array}$ & $\begin{array}{l}\text { Pressure } \\
\text { (MPA) }\end{array}$ & $\begin{array}{l}\text { Life - Theoretical } \\
\text { (No Of Cycles) }\end{array}$ & $\begin{array}{l}\text { Life - MATLAB } \\
\text { Simulation } \\
\text { (No Of Cycles) }\end{array}$ \\
\hline 1 & 0.05 & 69152 & 69132 \\
\hline 2 & 0.1 & 65484 & 65461 \\
\hline 3 & 0.15 & 62098 & 62074 \\
\hline 4 & 0.2 & 58969 & 58944 \\
\hline
\end{tabular}

\section{CONCLUSION}

The main of objective of this work is to estimate the fatigue life of bellows. This objective is achieved with the help of analytical and numerical approach for various pressure viz. $0.05 \mathrm{MPa}, 0.1 \mathrm{MPa}, 0.15 \mathrm{MPa}$ and $0.2 \mathrm{MPa}$ respectively. On comparison it is seen that fatigue life obtained by both approaches shows close match.

\section{REFERENCES}

[1] ASME, "ASME boiler and pressure vessel code-section VIII, division 1," in Appendix 26-Pressure Vessel and Heat Exchanger Joints, ASME, New York, NY, USA, 2000.

[2] EJMA, Standards of Expansion Joint Manufacturers Association, Expansion Joint Manufacturers Association, New York, NY, USA, 9th edition, 2008.

[3] H. Shaikh, G. George, and H. S. Khatak, "Failure analysis of an AM 350 steel bellows," Engineering Failure Analysis, vol. 8, no. 6, pp. 571-576, 2001.

[4] G. I. Broman, A. P. J"onsson, and M. P. Hermann, "Determining dynamic characteristics of bellows by manipulated beam finite elements of commercial software," International Journal of Pressure Vessels and Piping, vol. 77, no. 8, pp. 445-453, 2000.

[5] T. Li, "Effect of the elliptic degree of $\Omega$-shaped bellows toroid on its stresses," International Journal of Pressure Vessels and Piping, vol. 75, no. 13, pp. 951-954, 1998.

[6] C. Becht IV, "Fatigue of bellows, a new design approach," International Journal of Pressure Vessels and Piping, vol. 77 , no. 13, pp. 843-850, 2000.

[7] G. H. Faraji, M. M. Mashhadi, and V. Norouzifard, "Evaluation of effective parameters in metal bellows forming process,” Journal of Materials Processing Technology, vol. 209, no. 7, pp. 3431-3437, 2009.

[8] G. H. Faraji, M. K. Besharati, M.Mosavi, and H. Kashanizadeh ,"Experimental and finite element analysis of parameters in manufacturing of metal bellows," The International Journal of Advanced Manufacturing Technology, vol. 38, no. 7-8, pp. 641-648, 2008.

[9] B. H. Kang,M. Y. Lee, S.M. Shon, and Y. H.Moon, "Forming various shapes of tubular bellows using a single-step hydroforming process," Journal of Materials Processing Technology, vol. 193, no. 1-3, pp. 1-6, 2007.

[10] Subramanian. G., and Raghunandan, C., "On improving the fatigue life of U-form bellows," Journal of Materials Processing Technology, vol. 41,no.1, pp. 105-114, 1994.

[11] Jinbong Kim, "The effect of geometry on fatigue life for bellows" International Journal of Modern Physics , vol.6 pp. 343-348, 2012.

[12] Tingxin, L., Xiaoping, L., Tianxiang, L., Xigang, H., and Xinfeng, L., "Experimental research of toroid-shaped bellows behavior," International Journal of Pressure Vessels and Piping, vol. 63, no.2, pp. 141-146, 1995.

[13] Zhu, Y. Z., Wang, H. F., \& Sang, Z. F., "The effect of environmental medium on fatigue life for u-shaped bellows expansion joints,” International Journal of Fatigue, vol. 28, no.1, pp. 28-32, 2006.

[14] Pierce, S. O., \& Evans, J. L., "Failure analysis of a metal bellows flexible hose subjected to multiple pressure cycles," Engineering Failure Analysis, vol. 22, pp. 11-20, 2012.

[15] Koji K., Akio Y., Hideo C., Takeshi O., \& Mikio T., "Acoustic Emission For Fatigue Damage Detection Of Stainless Steel Bellows” Journal of Acoustic Emission, vol.23, pp.277-284, 2005. 